\title{
Sensory nerves directly promote osteoclastogenesis by secreting Cyp40
}

Guo-Xian Pei ( $\nabla$ nfperry@163.com )

Air Force Medical University Xijing Hospital: Xijing Hospital https://orcid.org/0000-0002-5089-5012

\section{Liu Yang}

Air Force Medical University Xijing Hospital: Xijing Hospital https://orcid.org/0000-0002-6498-4702

Junqin Li

Air Force Medical University

Bin Liu

General Hospital of Northern Theatre command

Hao Wu

Air Force Medical University Xijing Hospital: Xijing Hospital

\section{Shuaishuai Zhang}

Air Force Medical University Xijing Hospital: Xijing Hospital

\section{Zhuowen Liang}

Air Force Medical University Xijing Hospital: Xijing Hospital

\section{Shuo Guo}

Air Force Medical University Xijing Hospital: Xijing Hospital

\section{Guozhi Xiao}

Southern University of Science and Technology

\section{Ling Wang}

Southern University of Science and Technology

\section{Jing Xu}

Southern University of Science and Technology

\section{Huijie Jiang}

Xijing Hospital

\section{Pengzhen Cheng}

Air Force Medical University Xijing Hospital: Xijing Hospital

\section{Yue Song}

Air Force Medical University Xijing Hospital: Xijing Hospital

\section{Xing Lei}

Linyi People's Hospital

\section{Jimeng Wang}

Air Force Medical University Xijing Hospital: Xijing Hospital 
Air Force Medical University Xijing Hospital: Xijing Hospital

\section{Yi Gao}

Air Force Medical University Xijing Hospital: Xijing Hospital

Yang Liu

Air Force Medical University Xijing Hospital: Xijing Hospital

\section{Di Wang}

Air Force Medical University Xijing Hospital: Xijing Hospital

\section{Nazhi Zhan}

Southern University of Science and Technology

\section{Research}

Keywords: sensory nerves, osteoclastogenesis, cyp40, iTRAQ

Posted Date: November 23rd, 2021

DOI: https://doi.org/10.21203/rs.3.rs-1092196/v1

License: @ (i) This work is licensed under a Creative Commons Attribution 4.0 International License. Read Full License 

36

Title

Sensory nerves directly promote osteoclastogenesis by secreting Cyp40

\section{Authors}

Junqin $\mathrm{Li}^{1,2 \dagger}$, Bin Liu ${ }^{3 \dagger}$, Hao $\mathrm{Wu}^{1 \dagger}$, Shuaishuai Zhang ${ }^{1 \dagger}$, Zhuowen Liang ${ }^{1}$, Shuo Guo ${ }^{1}$, Huijie Jiang ${ }^{4}$, Yue Song ${ }^{1}$, Xing Lei ${ }^{5}$, Yi Gao ${ }^{1}$, Pengzhen Cheng ${ }^{1}$, Donglin $\mathrm{Li}^{6}$, Jimeng Wang $^{7}$, Yang Liu ${ }^{8}$, Di Wang ${ }^{1}$, Nazhi Zhan ${ }^{9}$, Guozhi Xiao ${ }^{10}$, Liu Yang ${ }^{1 *}$, GuoXian $\operatorname{Pei}^{1,2 *^{*}}$

\section{Affiliations}

1 Department of Orthopaedics, Xijing Hospital, Air Force Medical University, Xi'an 710032, China;

2 Southern University of Science and Technology Hospital, No. 6019 Liuxian Street, Xili Avenue, Nanshan District, Shenzhen, 518055, China;

3 Department of Orthopedics, General Hospital of Northern Theater Command, No .83, Wenhua Road, Shenhe District, Shenyang ,110016, China;

4 Lingtong Rehabilitation and Recuperation Center, Xi'an 710600, China;

5 Department of Orthopedics, Linyi people's hospital, LinYi 276000, China;

6 Northern Theater Air-Force Hospital of People's Liberation Army, Shenyang 110042, China;

7 Department of Orthopedics, 81 Army Hospital of the People's Liberation Army, Zhangjiakou 075000, China;

8 Department of Anaesthesiology and Perioperative Medicine, Xijing Hospital, Fourth Military Medical University, Xi'an 710032, China;

9 Department of Orthopedics, the Fifth Affiliated Hospital of Sun Yat-Sen University, Zhuhai Guangdong,519000, P.R.China;

10 Department of Biology and Guangdong Provincial Key Laboratory of Cell Microenvironment and Disease Research, Southern University of Science and Technology, Shenzhen 518055, China.

$\dagger, \ddagger$ These authors contributed equally to this work.

* Corresponding author. Email: nfperry@163.com (Guoxian Pei); yangliu@fmmu.edu.cn (Liu Yang) 37 
Abstract

\section{Background}

Given the afferent functions, sensors have been found exerting efferent influences and directly alter organ physiology ${ }^{1}$. sensory nerves have been found critical in osteoclasts and bone resorption ${ }^{2,3,4,5}$. However, the direct evidence of whether sensory nerve efferent influences osteoclast, remains lacking.

\section{Methods}

We treated mice with resiniferatoxin (RTX) or complete Freund's adjuvant (CFA) to induce sensory hypersensitivity. Bone histomorphometry including micro-ct, three-point bending assay, von kossa staining, calcein double labeling, toluidine blue staining, and trap staining were performed to monitor bone quality and bone cells. Multiple virus vectors were applied to trace signals between sensory nerves and osteoclasts. Sensory neurons (SN) and osteoclasts were cocultured to study the effects and mechanisms of the sensory nerves on osteoclasts in vitro. Isobaric tag for relative and absolute quantitation (iTRAQ) was used to identify secreted proteins in the sensory nerve.

\section{Results}

Here, we found sensory hypersensitivity significantly increased osteoclast bone resorption; SN directly promote osteoclastogenesis in vitro; and abundant sensory efferent signals transported into osteoclasts. Then our screening identified a novel neuropeptide Peptidyl-prolyl cis-trans isomerase D (Cyp40), is the reverse signal from the sensory nerve and plays a critical role for osteoclastogenesis, via aryl hydrocarbon receptor (AhR)-Ras/Raf-pErkNFATc1 pathway. The efferent signals from sensory nerves tend to involves in the rapid feedback process: vast majority of sensory efferent signals $(87.28 \%)$ present in fast-twitch myofibers.

\section{Conclusion}

This study revealed a novel mechanism of sensory nerves on osteoclasts: the direct promotion of osteoclastogenesis by the Cyp40. This mechanism may represent a direct, and quick response of sensory nerves to the changes in bone. Targeting the Cyp40 could therefore be a strategy to promote bone repair at the early stage of bone injury.

Key words: sensory nerves; osteoclastogenesis; cyp40; iTRAQ 


\section{Background}

In the tissues that are closely contacted with the external environment, such as skin, lung and gut, sensory nerves detect damaging stimuli, and can regulate the ensuing immune response by releasing neuropeptides seceretion ${ }^{6}$, 7,8 . However, other than the perception of pain, the role of sensory endings in the deep tissue such as the bone is not well understood.

Bones are innervated by a prolific network of neurochannels, around $77 \%$ of which consisted of sensory endings ${ }^{9}$. In bone, sensory endings closely contact with osteoblasts ${ }^{10}$, or osteoclasts ${ }^{11}$. Multiple studies have found sensory nerves have a direct effect on osteoblasts and bone formation ${ }^{12,13,14}$. A novel direct mechanism by which sensory nerves regulates HSC mobilization in bone marrow was also found recently ${ }^{9,15}$. Sensory nerves are critical in osteoclastogenesis, since patients with hereditary sensory neuropathy were reported to have no osteoclasts in the areas of severely degenerated sensory nerves ${ }^{16}$. There were also studies working on sensory neuropeptides, such as calcitonin gene related peptide (CGRP), and substance $\mathrm{P}$ (SP), influences on osteoclasts and bone resorption ${ }^{3,17}$. However, the direct evidence of whether sensory nerve efferent influences osteoclast, remains lacking.

To distinguish these possibilities, we treated mice with RTX or CFA to induce sensory hypersensitivity; applied multiple virus vectors to trace signals between sensory nerves and osteoclasts; as well as in vitro experiments to study the effects and mechanisms of the sensory nerves on osteoclasts.

\section{Materials and Methods}

\section{Animals and drug administration.}

Sprague-Dawley (SD) rats and Balb/c mice were obtained from the Experimental Animal Center of Air Force Medical University. GFP+ SD rats were purchased from Xing Ming Biomedical Technology Co., Ltd. (Shanghai, China). All animal procedures were approved by the Committee for the Care and Use of Laboratory Animals of Air Force Medical University and were performed in an authorized animal care facility.

RTX Three RTX (VIRTUE-CLARA, VTY25831) escalating doses (10 $\mu \mathrm{g}$ $\mathrm{kg}-1,20 \mu \mathrm{g} \mathrm{kg}-1$ and $30 \mu \mathrm{g} \mathrm{kg}-1)$ were injected subcutaneously into 4-weekold C57BL/6J mice on 3 consecutive days. Control littermates were injected with vehicle solution on the same days. 
CFA Rats were received intraplantar injections (i.pl.) of CFA (Sigma) or saline with the volume of $100 \mu \mathrm{l}$.

\section{Cells}

\section{BMSCs, BMMCs, and GFP+ BMMCs}

BMSCs ${ }^{18}$, BMMCs and GFP+ BMMCs were isolated from the femur bone marrow of 2-week-old WT and GFP+ SD rats as previously published.

\section{Sensory neurons}

SN were obtained from embryonic day 15 (E15) Sprague Dawley rat embryos using a published procedure ${ }^{19}$. Briefly, embryos were extracted from the uterine horns of pregnant rats. The spinal cords of the embryos were harvested to collect DRGs attached to the sides of the cord. The DRG explants were dissociated using $0.25 \%$ trypsin and purified by culturing the cells in media containing $10 \mu \mathrm{M}$ 5-fluoro-2-deoxyuridine (FdUrd) and $10 \mu \mathrm{M}$ uridine for 48 $\mathrm{h}$ to obtain SN.

\section{Lentiviral and plasmid vectors}

SN were transfected with overexpression plasmids or infected with lentiviral knockdown vectors, as previously described ${ }^{20}$. To overexpress Cyp40, SN were transfected with the pEGFP-N1 plasmid (Fig. S5) containing the gene that encodes Cyp40 (PPID; lv-Cyp40). The primer sequences used to detect lv-Cyp40 were: 5'-CGCAAATGGGCGGTAGGCGTG-3' and 5'CGTCGCCGTCCAGCTCGACCAG-3'. To knockdown Cyp40 and macrophage inhibitory factor (Mif), SN were infected with lentiviral vectors (GV298, U6-MCS-Ubiquitin-Cherry-IRES-puromycin) targeting Cyp40 (shCyp40) or Mif (shmif). The shCyp40 target sequence was CCTGCTAAAGGCTGTGATCAA and the shMif target sequence was CCTGCACAGCATCGGCAAGAT. For AhR knock down. AhRsiRNA (directed against rat AhR mRNA sequence [gi:6978474] from position 3291 to 3309): CGUUAGAUGUUCCUCUGUGTT (sense), and CACAGAGGAACAUCUAACGTT (antisense); with lentiviral vectors rLVU6-shRNA（AhR）-CMV-mCherry-2a-Puromycin. 
injected into the tibia for retrograde signal tracing (Retro); 1E+12v.g. PHP.SEGFP (Obio Technology, AG26973, pAAV-hSyn-hChR2(H134R)-EYFP) was injected into the tail vein for the detection of signals from peripheral nerves to cells; $700 \mathrm{nl} \mathrm{VSV-EGFP} \mathrm{(BrainVTA,} \mathrm{v01001)} \mathrm{were} \mathrm{injected} \mathrm{into} \mathrm{the}$ L3/L4 DRG.

\section{Trap staining}

To detect osteoclast differentiation in vitro, BMMCs were seeded into new dishes at $5.0 \times 10^{4}$ cells $/ \mathrm{mL}$. The cells were then co-cultured with $\mathrm{SN}$ or modified SN in which Cyp40 or Mif had been overexpressed or knocked down. Cells with no intervention (control group) or the cells that had been treated with saphenous nerve homogenate, recombinant Cyp40 or recombinant Mif were used. Cells were cultured in media containing M-CSF $(25 \mathrm{ng} / \mathrm{mL}$, Peprotech) for 3 days. Then different medium containing M-CSF ( $25 \mathrm{ng} / \mathrm{mL})$ and RANKL (100 ng/mL, Peprotech) used, accompanied by treatments (coculture, sensory nerve homogenate, recombinant proteins) for another 3 days. On day 6, cells were fixed and stained for tartrate-resistant acid phosphatase (Trap) using the leukocyte acid phosphatase kit (Sigma). Trap+ osteoclasts with more than three nuclei were quantified using ImageJ software.

\section{Osteoclast resorption activity}

Briefly, labeled FACS to CaP-coated plates, BMMCs cells were seeded into the coated plates in phenol red-free $\alpha$-MEM containing $25 \mathrm{ng} / \mathrm{mL}$ M-CSF for 3 days. Then different medium containing M-CSF and $150 \mathrm{ng} / \mathrm{mL}$ RANKL used, followed by treatments (co-culture, sensory nerve homogenate, recombinant proteins; see grouping strategy in Trap staining) for another 6 days. On day $9,100 \mu \mathrm{L}$ of the conditioned medium from each well was transferred to a new plate to measure resorbing activity using the bone resorption assay kit (Cosmobio). Fluorescence intensity was measured at an excitation wavelength of $485 \mathrm{~nm}$ and emission wavelength of $535 \mathrm{~nm}$.

\section{Quantitative real-time polymerase reaction chain (qPCR)}

Total RNA was purified from cells using TRIzol (Invitrogen, 15596026), reverse-transcribed using Prime Script TM RT Master Mix (TaKaRa, Japan) and subjected to qPCR using Taq SYBR Green Power PCR Master Mix (Invitrogen, A25777) on a CFX96TM real-time system (Bio-Rad). Gapdh was 
used as an internal control. The primer sequences were: Mmp9 forward: CGTCGTGATCCCCACTTACT and reverse: AACACACAGGGTTTGCCTTC; Ctsk forward: CAGTCCACAAGATTCTGGGG and reverse: GGTTCCTGTTGGGCTTTCAG and Gapdh forward: ATGTGTCCGTCGTGGATCTGA and reverse: ATGCCTGCTTCACCACCTTCTT.

\section{Immunofluorescent staining}

Femur specimens were dehydrated in 30\% sucrose and 10\% gum Arabic for 3 days at $4{ }^{\circ} \mathrm{C}$, embedded in optimal cutting temperature compound. Then, 10 $\mu \mathrm{m}$-thick sections were prepared. Immunofluorescent staining was performed according to standard protocols. Briefly, sections were permeabilized in $0.2 \%$ Triton X-100 (Sigma), nonspecific binding was blocked in 10\% donkey serum (Solarbio), and sections were incubated with primary antibodies against rat vWF (ab6994, Abcam, 1:200), TRAP (ab2391, Abcam, 1:200), BIII-tubulin (ab18207, Abcam, 1:2000), NF-H (ab8135, Abcam, 1:1000), CYP40 (127161-AP, Proteintech, 1:100), MIF (ab7202, Abcam, 1:250), CFL2 (sc-166958, Santa Cruz, 1:200), TPPP3 (sc-244483, Santa Cruz, 1:200), AHR (17840-1AP, Proteintech, 1:100),,TRPV1 (sc-398417, Santa Cruz, 1:200), Ctsk (sc48353, Santa Cruz, 1:100), Osx (ab209484, abcam, 1:1000), CD45 (NB10077417SS, Novus, 1:100), or Mtsn (sc-13122, Santa Cruz, 1:100) overnight at $4{ }^{\circ} \mathrm{C}$. Fluorescent-conjugated secondary antibodies were used to detect fluorescent signals, followed by counterstaining with Hoechst 33342 (Sigma Aldrich, 1000x). Images of the center field of view were captured for each independent sample using a confocal microscope (A1R, Nikon) and immunofluorescent staining intensity was quantified using Image J software.

\section{Western blotting}

Western blots were performed according to standard protocols. The primary antibodies were rat CYP40 (12716-1-AP, Proteintech, 1:500), RAS (ab52939, Abcam, 1:5000), C-RAF (ab50858, Abcam, 1:1000), ERK (ab17942, Abcam, 1:1000), P-ERK (ab201015, Abcam, 1:1000), MIF (ab7202, Abcam, 1:2000), AHR (17840-1-AP, Proteintech, 1:500), CYP1A1 (13241-1-AP, Proteintech, 1:500), eGFP (CAB4211, Invitrogen, 1:200), CD9 (ab92726, Abcam, 1:2000), GAPDH (ab9485, Abcam, 1:2500) and $\beta$-actin (ab8226, Abcam, 1:1000). 
Saphenous nerves from $300 \mathrm{Balb} / \mathrm{c}$ mice were randomly divided into 2 groups (150 mice/group). The samples in the first group were rinsed to wash away most axoplasmic proteins (Y1). The samples in the other group were untreated (Y2). More abundant components in the Y2 group were considered axoplasmic proteins. iTRAQ targeting sensory nerves were performed by Beijing Genomics institution as published before. ${ }^{21}$ Proteins from each sample were labeled with iTRAQ reagent (Applied Biosystems) as follows: sample Y1 - 119 tags, Sample Y2 - 121 tags. Proteins with P-values $<0.05$ and fold changes $>1.2$ between groups were considered differentially expressed proteins.

Alteration of differentially secreted proteins after CFA treatment were also detected by iTRAQ,. 20 SD rats were injected with CFA, and randomly divided into 2 groups (10 rats/group): untreated group (CFA-whole), and the group which has been rinsed to wash away most axoplasmic proteins (CFAstructure). Control littermates (20 SD rats) were injected with saline on the same day; and were grouped in the same way: 10 rats/group, Ctrl-whole and Ctrl-Structure.

\section{Immunoelectron Microscopy (IEM)}

Femurs seeded with BMMCs were isolated co-cultured with SN transfected with plasmids containing EGFP-tagged Cyp40. M-CSF was added to the media for the first $24 \mathrm{~h}$ to induce BMMC attachment. M-CSF and RANKL were then added to the media to induce differentiation of BMMCs into osteoclasts for 5 days. The osteoclast-bone composite were fixed in $4 \%$ paraformaldehyde and $0.05 \%$ glutaraldehyde for $24 \mathrm{~h}$ and decalcified in $10 \%$ EDTA for 4 weeks and sectioned using a vibratome (VT1000S, Leica) at 45 $\mu \mathrm{m}$. Sections were washed 30 times with $0.01 \mathrm{M}$ PBS to remove fixative, blocked in 5\% BSA and $0.05 \%$ Triton for $3 \mathrm{~h}$, washed 15 times with PBS, incubated with primary antibody against Cyp40 (12716-1-AP, Proteintech, 1:100) or EGFP (ab6556, Abcam, 1:1000) diluted in 1\% BSA and 0.05\% Triton $\mathrm{X}$ for $24 \mathrm{~h}$ at room temperature, and washed 30 times with PBS. The sections were incubated with $1.4 \mathrm{~nm}$ nanogold-IgG goat anti-rabbit IgG antibody (\#2003-1, Nanoprobes, 1:100) diluted in 1\% BSA and 0.05\% Triton for $4 \mathrm{~h}$ at room temperature, washed 35 times with PBS, fixed in $20 \mathrm{~mL} / \mathrm{L}$ glutaraldehyde for $20 \mathrm{~min}$, and then washed 30 times in PBS and then deionized water. Sections were incubated in HQ Silver Enhancement Kit (\#2012-45, Nanoprobes) for $15 \mathrm{~min}$ in the dark to enhance sensitivity. The reaction was stopped in cold deionized water. Sections were then washed 30 times in cold deionized water, washed 30 times in phosphate buffer (PB), 
fixed in $5 \mathrm{~mL} / \mathrm{L}$ citric acid for $1.5 \mathrm{~h}$, washed with PB. The washed sections were dehydrated in ethanol gradient from $300 \mathrm{~mL} / \mathrm{L}$ to $1000 \mathrm{~mL} / \mathrm{L}$ immersed in acetone: Epon812 (1:1) for $45 \mathrm{~min}$, immersed in Epon812 for $12 \mathrm{~h}$, and then flat embedded and polymerized at $60{ }^{\circ} \mathrm{C}$ for $24 \mathrm{~h}$. Ultrathin sections were then obtained using an ultramicrotome (EM UC6, Leica) and stained with uranyl acetate and lead citrate. Transmission electron microscope images were captured and analyzed using a JEM-1230 (JEOL) with Gatan Digital Micrograph 3.9.

\section{Exosomes}

Medium was collected from the co-culture system and centrifuged at $500 \mathrm{~g}$ for $5 \mathrm{~min}$ to remove cellular components. This was followed by $2000 \mathrm{~g}$ centrifugation for $10 \mathrm{~min}$ to remove cellular debris, and another centrifugation at $10,000 \mathrm{~g}$ for $30 \mathrm{~min}$ to remove large particle particles. The supernatant was filtered through a $0.22 \mu \mathrm{m}$ filter, centrifuged at $100,000 \mathrm{~g}$ for $70 \mathrm{~min}$, and the (non-exosomal) supernatant was collected (MS). The exosomes in the pellet were resuspended in PBS and transferred to a new centrifuge tube.

\section{Co-immunoprecipitation}

The cells were lysed in IP lysis buffer (Thermo Scientific, \#87787) for 1 hour, incubated with PureProteome Protein A or Protein G Magnetic Beads (Millipore, \#LSKMAGA02) and antibodies against AHR (17840-1-AP, Proteintech, 1:50) and EGFP (ab6556, Abcam, 1:100) at $4{ }^{\circ} \mathrm{C}$ overnight. The immunoprecipitates were subjected to immunoblotting.

\section{Three-point bending tests}

To measure the bone strength of the femurs, the BOSE Electroforce (3220) was used to perform the three-point bending test. Place the femur specimen steadily on the bending jig so that the short axis of the femur is consistent with the direction of the force. The span of the two fulcrums is $8 \mathrm{~mm}$, the preload is $0.5 \mathrm{~N}$, and the loading speed is $0.02 \mathrm{~mm} / \mathrm{s}$, The route is $2 \mathrm{~mm}$, and the test is terminated after the specimen is destroyed. The biomechanical measurement data were collected from the load-deformation curves. The maximum load $(\mathrm{N})$ was recorded. 


\section{Biochemical parameters}

Detection of biochemical parameters in serum were performed by automatic biochemical analyzer (MS-480) and matching kits; including total inorganic phosphate (P, 201SJTZ306), cholesterol (TCH, 201SJTZ202), triglycerides (TG, 201SJTZ201), Glucose (Gluhk, 201SJTZ108), creatine kinase (CK, 201SJTZ006), Urea Nitrogen (Urea, 201SJTZ106), Creatinine (CR, 201SJTZ105), and Uric acid (UA, 201SJTZ107).

\section{Results}

\section{Sensory hypersensitivity induces osteopenia in mice}

To investigate whether sensory nerve exert efferent influences on bone homeostasis, RTX were injected in mice to induce sensory hypersensitivity (Fig 1a). Microcomputed tomography $(\mu \mathrm{CT})$ analysis of tibia revealed that key parameters, including bone volume/tissue volume fraction (BV/TV), trabecular number (Tb.N), trabecular separation (Tb.Sp), and bone mineral density (BMD), were significantly altered in sensory hypersensitivity mice compared with their control littermates (Fig 1b). However, trabecular thickness (Tb.Th), trabecular bone surface/bone volume (Tb.BS/BV), and all key parameters in cortical bone were not significantly altered (Fig.S1a). Three-Point Bending Test also showed the decrease of bone quality (Fig 1c). Serum biochemical analyses showed a dramatic reduction of serum inorganic phosphorus (P, Fig. 1d). Since the serum $\mathrm{P}$ abnormal were always induced by renal dysfunction, we tested serum biochemical makers (Fig.S1c) related to renal function, and found all serum renal markers were not changed in RTX mice. Our results above have hinted the efferent influence of sensory nerve on bone homeostasis.

Von Kossa staining showed decreased osteoid in RTX treat mice (Fig 1e). Calcine double labeling confirmed the reduced bone formation and mineral apposition rate (Fig 1f). Toluidine blue staining showed decreased osteoblast function and bone formation in RTX mice (Fig 1g). Accordingly, the serum level of procollagen type N-terminalpropeptide (P1NP, Fig 1h) was significantly decreased. All these were consistent with our published before, that is sensory nerve exert direct efferent regulation on bone marrow stem cells (BMSC) differentiation ${ }^{14}$. Then we found the serum levels of collagen type I cross-linked C-telopeptide (CTX), an osteoclast bone resorption marker, were significantly elevated in RTX treated mice compared with control littermates (Fig 1h).

In order to further dissect the mechanism of sensory nerve action, we purified sensory neuron (SN) from the dorsal root ganglion (DRG, Fig. S1.d). The SN were isolated co-cultured with osteoclasts such that direct cell contacts were avoided (Fig. 1.i). The presence of SN significantly increased the number of 
osteoclasts (Fig 1j), osteoclast resorption activity (Fig $1 \mathrm{k}$ ), and resorption related genes (Fig 1 1). The results above have hinted the direct promotion of sensory nerve on osteoclastogenesis.

\section{Abundant signals in sensory nerves efferent transported into cells in bone}

Reverse signal from sensory nerve to bone has been suggested but was not generally accepted ${ }^{1}$. In this study we applied engineered virus vectors, including adeno-associated virus (AAV), and vesicular stomatitis virus (VSV) to trace signals between sensory nerve and bone cells.

Firstly, we injected AAV2-EGFP/Retro in bone to trace if the vectors from bone trace the signal specifically, instead contaminate cells broadly. AAV2EGFP/Retro, engineered by in vivo directed evolution, permits retrograde access to projection neurons ${ }^{22,23}$. We used AAV2-EGFP/Retro retrograde tracing in mice and found that sensory endings received direct inputs from in bone, the EGFP indicator specifically distributed in the outer circle and dotted in the central part of the DRG (Fig 2a, b), indicating that the viral vector can track the signal between the sensory nerve and the peripheral organs.

Also, we injected anterograde VSV-EGFP locally into DRG (Fig 2c, and Fig S.2a). VSV were designed to specifically infected neurons, and for anterograde transsynaptic tracing ${ }^{24}$. Abundant signals were found in osteoclasts (cathepsin $\mathrm{K}, \mathrm{Ctsk}^{+}$), osteoblastic cells (osterix, $\mathrm{Osx}^{+}$), and leukocytes (cluster of differentiation $45, \mathrm{CD}^{+} 5^{+}$) in bone (Fig $2 \mathrm{~d}$ ). In all bone cells, signals from sensory nerves are concentrated in the cytoplasm, and only a small amount is transported into the nucleus (Fig $2 \mathrm{e}, \mathrm{f}$ ). Many proteins in the nucleus are transcription factors which enter the nucleus and rebind to the chromatin to initiate the transcriptional process. This regulation is relatively a slow process. On the contrary, the proteins in the cytoplasm perform functions such as kinases or adaptors, which is a faster regulation. After entering muscles, most of the factors (87.28\%) secreted by sensory nerves are present in fast-twitch myofibers, which display rapid bursts of contraction, and fatigue rapidly. However, only a small amount of proteins $(12.72 \%)$ is distributed in slow-twitch myofibers which exert slow contractions (Fig $\mathrm{S} 4)^{25}$. The above results suggest that the efferent function of sensory nerves is tend to involved in the rapid adjustment process.

PHP.S-EGFP vectors were AAV vectors designed for transfer gene to cells of the peripheral nervous systems (PNS), so its target cells limited to the peripheral nervous system. Further, we used PHP.S virus to compare signals in the bone cells from PNS and from sensory nerve (Fig 2g, and Fig 2c-f). Our results showed that the PHP.S-EGFP signal also enters various cells in the bone (Fig 2h). But we did not find significant difference in the 
distribution of signals from sensory nerves and peripheral nerves into osteoclasts (Fig 2i). This suggests that the regulation of bone by sensory nerves is a common process.

\section{Cyp40 is crucial in sensory nerve elevating osteoclastogenesis}

To search for key factors through which sensory nerve elevates osteoclastogenesis, we screened the neuropeptides in sensory nerve axons. Since the functional neuropeptides synthesized in the dorsal root ganglion are transported peripherally and stored in the axon, before being released to target cells ${ }^{26}$. Isobaric tag for relative and absolute quantitation (iTRAQ) was used to identify proteins in saphenous nerve axoplasm (Fig 3a). The technique uses a variety of isotope reagents to label the $\mathrm{N}$-terminal or lysine side chain groups of protein polypeptides, and then the labeled peptides are analyzed by a high-precision mass spectrometer. This allows for the identification of multiple proteins and provides reliable quantitative proteome information ${ }^{27}$.

A total of 237 proteins were identified in saphenous nerve axoplasm (Fig 3b). Functional classification of these axoplasmic proteins using Clusters of Orthologous Groups (COG) (Fig 3c) revealed that one-quarter (or 24.5\%) of these proteins are involved in posttranslational modifications, protein turnover or are chaperones. Our previous in vitro experiments (Fig. 1i-1) indicated that the sensory nerves mainly increased osteoclastic differentiation. Indeed, we identified 17 axoplasm components related to cell proliferation and differentiation (Fig 3d). Some of axoplasm components (Cyp40, Mif, cofilin 2 (Cfl2), tubulin polymerization promoting protein family member 3 (Tppp3)) were confirmed by immunofluorescent staining, and Cyp40 and Mif were detected in axons (Fig 3e). Osteoclasts and many immune cells are differentiated from HSC. We screened the relevant literature and found that the Cyp $40^{28,29}$ and $\mathrm{Mif}^{30,31}$ are closely related to immune cells. Then, Mif and Cyp40 were selected for subsequent studies.

Both of Cyp40 and Mif increase osteoclastic differentiation (Fig $3 \mathrm{f}, \mathrm{g}$ ) and resorption activity of osteoclasts (Fig $3 \mathrm{~h}$, i). To further study the roles of the two factors in osteoclastogenesis promotion by sensory nerve, we downregulated Cyp40 (shCyp40) and Mif (shmif) in SN (Fig S3 a, b). Osteoclasts were then cultured with the modified SN. Downregulation of Cyp40 significantly attenuated the ability of the SN to promote osteoclastogenesis. However, downregulation of Mif did not significantly affect the ability of SN to promote osteoclastogenesis (Fig. $3 \mathrm{j}$-m). Thus, we concluded that Cyp40 is crucial in the ability of SN to promote osteoclastogenesis in vitro.

Further, we screened the instant alteration of secrete proteome after sensory hypersensitivity. We applied CFA to induce sensory hypersensitivity (Fig 
S4.a), and found 272 secreted proteins changed 48 hours after CFA injection (Fig S4.b). KEGG enrichment of differentially proteins showed top 15 pathways. Among them, proteins associated with tight junction showed the most prominent difference (Fig S4.c). Notably, Cyp40 were downregulated by CFA (Fig S4.d).

\section{Cyp40 is a neuropeptide, enters osteoclasts by transmembrane, downregulates Ras/c-Raf/p-Erk to promote osteoclastogenesis}

Back in vivo, we found Cyp40 level was upregulated in the RTX treat mice (Fig 4a), including serum Cyp40 level (Fig 4b); the recombinant Cyp40 dramatic reduced osteoblastic differentiation (Fig S3 d); these results confirmed the crucial role of Cyp40 in the sensory nerve efferent functions. Then we constructed and transfected an EGFP-tagged Cyp40 vector into SN to verify if Cyp40 is released from SN into osteoclasts (Fig S3 a, b). Coculturing the modified SN with osteoclasts led to the detection of EGFPtagged Cyp40 in osteoclasts (Fig 4c), indicating Cyp40 was secreted by SN and taken up by osteoclasts. ELISA results show a dose-response curve of Cyp40 in response to increasing numbers of SN (Fig 4d). These results therefore prove that Cyp40 is a secreted factor from $\mathrm{SN}$.

The transportation of Cyp40 has not yet been studied ${ }^{32}$. Then we traced the transportation of Cyp40 between cells. In the co-culture medium, Cyp40 was found outside exosomes (Fig 4e), indicating Cyp40 was not transported between SN and osteoclasts via exosomes. IEM that targets Cyp40 shows the factor entering osteoclasts through the cell membrane (Fig 4f, II, and Fig 4g; red arrow), instead wrapped by membrane (Fig 4f, II, and Fig 4g; green arrow). Since Cyp40 is abundant in neurons, we detected its traffic also in the brain. Cyp40 is transported between neurons in the brain also through the cell membrane (Fig 4g, red arrow). As shown in the figures, Cyp40 was crossing the cell membrane (g 4f, II, and Fig 4g, red arrow). After entering osteoclasts, Cyp40 became widely distributed in the cells. It was present at low levels in the nucleus (Fig 4f, III) and higher levels throughout the cytoplasm (Fig 4f, I), including the ruffled border responsible for bone resorption (Fig 4d, IV). Thus, Cyp40 secreted from SN enters osteoclasts via a non-exosomal mechanism to promote osteoclastogenesis.

Ras/c-Raf/p-Erk signaling has been implicated in osteoclast survival, proliferation, apoptosis, formation, polarity, and differentiation ${ }^{33}$. Thus, we studied the effects of SN on ERK signaling in osteoclasts. We found that SN downregulated the expression of Ras and c-Raf, and phosphorylation of ERK in osteoclasts. Cyp40 also downregulated Ras/c-Raf/p-Erk. The downregulation of Ras/c-Raf/p-Erk by SN was bolished when Cyp40 was knocked down in SN. Moreover, Ras/c-Raf/p-Erk were also downregulated in the RTX treat mice, when Cyp40 was upregulated in DRG from the same 
mice (Fig 4i). These results indicates that Cyp40 plays a crucial role in the ability of sensory nerves to negatively regulate Ras/c-Raf/p-Erk in osteoclasts (Fig 4h). P-ERK can inhibit osteoclastogenesis by downregulating NFATc $^{14,35}$. NFATc1 is a key transcription regulator in osteoclasts ${ }^{36}$. We found an increase in NFATc1 mRNA expression level and simultaneous increases in osteoclastogenesis when p-Erk was downregulated in osteoclasts (Fig 4j). Moreover, these changes in NFATc1 expression were dependent on Cyp40 (Fig 4j). Overall, these results suggest that the sensory nerves promote osteoclastogenesis by secreting Cyp40. Secreted Cyp40 downregulates Ras/c-Raf/p-Erk, which releases the inhibition of NFATc1 by p-Erk and promotes osteoclastogenesis.

\section{Sensory nerve and its Cyp40 promote osteoclastogenesis by AhR}

AhR activates Ras, which in turn activates ERK and promotes cell proliferation and differentiation ${ }^{37}$. Cyp40 has been reported to modulate expression and distribution of $\mathrm{AhR}^{38}$. Thus, we examined if AhR is involved in the ability of sensory nerves to promote osteoclastogenesis. Firstly, we found a decrease of AhR in RTX treat mice (Fig 4i). Then, we found an interaction between AhR in osteoclasts and Cyp40 from SN (Fig 5a).

Furthermore, western blotting showed that co-culture with SN reduced the expression of AhR in osteoclasts; and the AhR downregulation was attenuated by knocking down Cyp40 in SN (Fig 5b). Above results indicate Cyp40 binds to and downregulates AhR in osteoclasts.

Then, we found that AhR knock down in osteoclast dramatic decreased osteoclastogenesis, and significantly attenuated sensory efferent promotion on osteoclastogenesis (Fig 5c). Moreover, Ras/c-Raf/p-Erk level was also decreased after AhR knock down in osteoclasts (Fig 5d).

AhR exerts canonical transcription activity in the nucleus and transcriptionindependent protein activity in the cytoplasm ${ }^{39}$. Immunofluorescent staining of osteoclasts showed that AhR mainly co-localized with neuron-derived Cyp40 in the cytoplasm (Fig5 e, f). Furthermore, the promotion of osteoclastogenesis by SN and Cyp40 did not involve changes in the expression of cytochrome P450 1A1 (Cyp1a1), a marker of canonical AhR transcriptional activity (Fig 5g). Collectively, these findings suggest that Cyp40 binds, and down-regulates AhR, to decrease Ras/c-Raf/p-Erk level, and finally promote osteoclastogenesis.

\section{Discussion}

Sensory endings enable the rapid detection of environmental insults -for example- cold, heat or pain-to avoid environmental damage ${ }^{40,41}$; and the sensory nerves are integral for the generation of immune responses to protect 
body intact ${ }^{42}$. Given the afferent functions, sensors have been found exerting efferent influences and directly alter organ physiology ${ }^{1}$. Gao X et al. found sensory nerves directly regulate HSC maintenance and egress from the bone marrow ${ }^{42}$. Similar functions of sensory nerves have been reported in dendritic cells in skin infection ${ }^{7}, \mathrm{CD}^{+}$and resident innate lymphoid type 2 cells in airway inflammation ${ }^{8}$. Notably, T lymphocytes, dendritic cells, and osteoclasts are all derived from HSC. Here in this study, we found sensory hypersensitivity significantly increased osteoclast bone resorption; SN directly promote osteoclastogenesis in vitro co-culture system; and abundant signals efferent transported from sensory nerves into osteoclasts. All results above suggest the direct promotion of sensory nerves on osteoclastogenesis.

In the muscle tissue, which are consisted of fast-twitch myofibers responsible for rapid bursts of contraction, and slow-twitch myofibers which exert slow contractions for endurance exercises such as standing; vast majority of sensory efferent signals $(87.28 \%)$ present in fast-twitch myofibers. The above results suggest that the efferent function of sensory nerves tend to involves in the rapid feedback process. Zhu et al. found that sensory nerves and osteoclasts were increased in subchondral bone as early as the 1st week after anterior cruciate ligament transection (ACLT) surgery, that is at the early stage of osteoarthritis $(\mathrm{OA})^{43}$.

However, Hao et $\mathrm{al}^{10}$. found that the number of osteoclasts were not altered in mouse models of sensory denervation (TrkA Avil-/- or adult iDTR Avil $\mathrm{fl}_{\text {-/- }}$ mice injected with $1 \mu \mathrm{g} / \mathrm{kg}$ diphtheria toxin). Toru et al. ${ }^{44}$ also observed no significant changes in osteoclasts in Sema3a synapsin-/- mice even though the number of sensory innervations in trabecular bone were significantly decreased. The difference between these reports and our results may be due to the sensory denervation induce compensation of other nerves, which also regulate osteoclasts. For example, patients with hereditary sensory neuropathy have no osteoclasts in the areas of severely degenerated sensory nerves ${ }^{16}$. However, patients with familial dysautonomia, which is characterized by autonomic and sensory dysfunction ${ }^{45}$, suffer osteoporosis ${ }^{46}$. Neural regulations of bone are indeed complicated. The factors that trigger sensory nerves to directly promote osteoclastogenesis, need further investigation.

Then our screening identified a novel neuropeptide Cyp40, is the reverse signal from the sensory nerve and plays a critical role for osteoclastgenesis. Cyp40 is a rarely studied protein. Most of the researches on Cyp40 focus on its features as chaperone which regulates the function of hormone-like receptors such as steroid receptors ${ }^{47,48,49,50,51,52,53}$, estrogen receptor ${ }^{54,55}$ and $\mathrm{AhR}^{38,56}$, to participate in the stress responses $57,58,59,60,61$. It has never been reported to function as a neuropeptide, and its extracellular transportation has not been investigated. Importantly, the roles of Cyp40 in bone have also not yet been studied. Here we found Cyp40 was not 
transported via exosomes. The factor enters osteoclasts by directly passing

\section{List of abbreviations}

\begin{tabular}{|l|l|}
\hline Resiniferatoxin & RTX \\
\hline Complete Freund's adjuvant & CFA \\
\hline Sensory neuron & SN \\
\hline Isobaric tag for relative and absolute quantitation & iTRAQ \\
\hline Peptidyl-prolyl cis-trans isomerase D & Cyp40 \\
\hline Calcitonin gene related peptide & CGRP \\
\hline Macrophage inhibitory factor & Mif \\
\hline Cofilin 2 & Cf12 \\
\hline Tubulin polymerization promoting protein family member 3 & Tppp3 \\
\hline
\end{tabular}

After entering osteoclasts, Cyp40 was found to downregulate Ras/c-Raf/pErk signal by AhR. AhR exerts dual effects as it can promote or inhibit osteoclasts $^{62,63}$. Canonical AhR activity has been shown to promote osteoclastogenesis $^{64}$. Ye et al. recently revealed a non-canonical transcription-independent function of the $\mathrm{AhR}^{39}$, which has not yet been studied in bone. Here, we found that the resulting downregulation of AhR by Cyp40 secreted from the sensory neuron did not involve canonical AhR activity. The non-canonical activity of AhR regulates ERK signaling ${ }^{39} \cdot \mathrm{p}$-Erk enters the nucleus to regulate the expression of NFATc1, a master transcription factor that regulates multiple osteoclast-specific genes including Ctsk and matrix metallopeptidase 9 (MMP9) ${ }^{36}$, to promote ${ }^{65,66}$ or inhibit $^{34,35}$ osteoclastogenesis. In our co-culture system in which sensory nerves and its Cyp40 promoted osteoclastogenesis, NFATc1 and its downstream target genes, Ctsk and Mmp9 were upregulated while AhR/Ras/c-Raf/p-Erk was downregulated.

\section{Conclusions}

In summary, sensory nerves secret Cyp40, which enters osteoclasts, binds to, and downregulates AhR and Ras/c-Raf/p-Erk. In turn, this promotes the inhibition of NFATc1 by p-Erk and upregulates the MMP9 and Ctsk, consequently promoting osteoclastogenesis. Our findings on Cyp40 indicate that the factor is a new target in sensory nerve-bone research. 
Science Foundation of China (Grant No. 81772377). Author contributions: Junqin Li, Bin Liu, Hao Wu, Shuaishuai Zhang designed and performed the main study experiments, analyzed the data, and wrote the manuscript; Zhuowen Liang purified SN, and modified SN; Shuo Guo and Yi Gao performed western blotting experiments; Di Wang and Yang Liu performed PCR experiments; Huijie Jiang, Yue Song, Xing Lei, and Pengzhen Cheng performed innervated-TEBG surgeries; Donglin Li and Jimeng Wang performed innervated-FDBD surgeries; Guozhi Xiao commented on and approved the final manuscript; Guoxian Pei and Liu Yang conceived and supervised the research. Competing interests: The authors declare no competing interests. Data and materials availability: All data needed to evaluate the conclusions in the paper are present in the paper and/or the Supplementary Materials. Additional data related to this paper may be requested from the authors.

\section{References}

1. Pavlov VA, Chavan SS, Tracey KJ. Molecular and Functional Neuroscience in Immunity. Annual review of immunology 36, 783-812 (2018).

2. He H, et al. CGRP may regulate bone metabolism through stimulating osteoblast differentiation and inhibiting osteoclast formation. Mol Med Rep 13, 3977-3984 (2016).

3. Ishizuka K, Hirukawa K, Nakamura H, Togari A. Inhibitory effect of CGRP on osteoclast formation by mouse bone marrow cells treated with isoproterenol. Neurosci Lett 379, 47-51 (2005).

4. Liu D, Jiang LS, Dai LY. Substance P and its receptors in bone metabolism. Neuropeptides 41, 271-283 (2007).

5. Fukuda A, et al. Hemokinin-1 competitively inhibits substance P-induced stimulation of osteoclast formation and function. Neuropeptides 47, 251-259 (2013).

6. Michoud F, et al. Epineural optogenetic activation of nociceptors initiates and amplifies inflammation. Nature biotechnology.

7. Kashem SW, Riedl MS, Yao C, Honda CN, Vulchanova L, Kaplan DH. Nociceptive Sensory Fibers Drive Interleukin-23 Production from CD301b+ Dermal Dendritic Cells and Drive Protective Cutaneous Immunity. Immunity 43, 515-526.

8. Talbot S, et al. Silencing Nociceptor Neurons Reduces Allergic Airway Inflammation. Neuron 87, 341-354. 
9. Gao X, Zhang D, Xu C, Li H, Caron KM, Frenette PS. Nociceptive nerves regulate haematopoietic stem cell mobilization. Nature, (2020).

10. Chen H, et al. Prostaglandin E2 mediates sensory nerve regulation of bone homeostasis. Nature communications 10, 181 (2019).

11. Hara-Irie F, Amizuka N, Ozawa H. Immunohistochemical and ultrastructural localization of CGRP-positive nerve fibers at the epiphyseal trabecules facing the growth plate of rat femurs. Bone 18, 29-39 (1996).

12. Zhang PX, Jiang XR, Wang L, Chen FM, Xu L, Huang F. Dorsal root ganglion neurons promote proliferation and osteogenic differentiation of bone marrow mesenchymal stem cells. Neural regeneration research 10, 119-123 (2015).

13. Silva DI, Santos BPD, Leng J, Oliveira H, Amédée J. Dorsal root ganglion neurons regulate the transcriptional and translational programs of osteoblast differentiation in a microfluidic platform. Cell death \& disease 8, 3209 (2017).

14. Zhang S, et al. Dorsal Root Ganglion Maintains Stemness of Bone Marrow Mesenchymal Stem Cells by Enhancing Autophagy through the AMPK/mTOR Pathway in a Coculture System. Stem cells international 2018, 8478953 (2018).

15. Tikhonova AN, Aifantis I. Pain-sensing neurons mobilize blood stem cells from bone marrow. Nature, (2020).

16. Teot L, Arnal F, Humeau C, Pous JG, Dimeglio A. Ultrastructural aspects of nerves, bones, and vessels in hereditary sensory neuropathy. Journal of orthopaedic research : official publication of the Orthopaedic Research Society 3, 226-235 (1985).

17. Offley SC, et al. Capsaicin-sensitive sensory neurons contribute to the maintenance of trabecular bone integrity. Journal of bone and mineral research : the official journal of the American Society for Bone and Mineral Research 20, 257-267 (2005).

18. Matsushita Y, et al. A Wnt-mediated transformation of the bone marrow stromal cell identity orchestrates skeletal regeneration. Nature communications 11, 332 (2020).

19. Burkey TH, Hingtgen CM, Vasko MR. Isolation and culture of sensory neurons from the dorsal-root ganglia of embryonic or adult rats. Methods in molecular medicine 99, 189-202 (2004).

20. Heffernan C, Maurel P. Lentiviral Transduction of Rat Schwann Cells and Dorsal Root Ganglia Neurons for In Vitro Myelination Studies. Methods in molecular biology (Clifton, NJ) 1739, 177-193 (2018). 
21. Discovery of potential colorectal cancer serum biomarkers through quantitative proteomics on the colonic tissue interstitial fluids from the AOM-DSS mouse model. Journal of Proteomics 132, 31-40 (2016).

22. Ren S, et al. The paraventricular thalamus is a critical thalamic area for wakefulness. Science (New York, NY) 362, 429-434 (2018).

23. Tervo DG, et al. A Designer AAV Variant Permits Efficient Retrograde Access to Projection Neurons. Neuron 92, 372-382 (2016).

24. Beier KT, et al. Anterograde or retrograde transsynaptic labeling of CNS neurons with vesicular stomatitis virus vectors. Proceedings of the National Academy of Sciences of the United States of America 108, 15414-15419 (2011).

25. Flynn JM, Meadows E, Fiorotto M, Klein WH. Myogenin Regulates Exercise Capacity and Skeletal Muscle Metabolism in the Adult Mouse. PLoS ONE 5, e13535 (2010).

26. Carr R, Frings S. Neuropeptides in sensory signal processing. Cell and tissue research 375, 217-225 (2019).

27. Sun H, et al. Proteomic analysis of amino acid metabolism differences between wild and cultivated Panax ginseng. Journal of ginseng research 40, 113-120 (2016).

28. Park MS, Chu F, Xie J, Wang Y, Bhattacharya P, Chan WK. Identification of cyclophilin-40interacting proteins reveals potential cellular function of cyclophilin-40. Analytical biochemistry 410, 257-265 (2011).

29. Pratt WB, Toft DO. Steroid receptor interactions with heat shock protein and immunophilin chaperones. Endocrine reviews 18, 306-360 (1997).

30. Calandra T, Bucala R. Macrophage Migration Inhibitory Factor (MIF): A Glucocorticoid Counter-Regulator within the Immune System. Critical reviews in immunology 37, 359-370 (2017).

31. Kang I, Bucala R. The immunobiology of MIF: function, genetics and prospects for precision medicine. Nature reviews Rheumatology 15, 427-437 (2019).

32. Schumann M, et al. Identification of low abundance cyclophilins in human plasma.

Proteomics 16, 2815-2826 (2016).

33. Lee K, Seo I, Choi MH, Jeong D. Roles of Mitogen-Activated Protein Kinases in Osteoclast Biology. International journal of molecular sciences 19, (2018). 
34. Lee EG, Yun HJ, Lee SI, Yoo WH. Ethyl acetate fraction from Cudrania tricuspidata inhibits IL-1beta-stimulated osteoclast differentiation through downregulation of MAPKs, c-Fos and NFATc1. The Korean journal of internal medicine 25, 93-100 (2010).

35. Kim B, Lee KY, Park B. Icariin abrogates osteoclast formation through the regulation of the RANKL-mediated TRAF6/NF-kappaB/ERK signaling pathway in Raw264.7 cells. Phytomedicine : international journal of phytotherapy and phytopharmacology 51, 181-190 (2018).

36. Negishi-Koga T, Takayanagi H. Ca2+-NFATc1 signaling is an essential axis of osteoclast differentiation. Immunological reviews 231, 241-256 (2009).

37. Pierre S, et al. Induction of the Ras activator Son of Sevenless 1 by environmental pollutants mediates their effects on cellular proliferation. Biochemical pharmacology 81, 304-313 (2011).

38. Luu TC, Bhattacharya P, Chan WK. Cyclophilin-40 has a cellular role in the aryl hydrocarbon receptor signaling. FEBS letters 582, 3167-3173 (2008).

39. Ye M, et al. Activation of the Aryl Hydrocarbon Receptor Leads to Resistance to EGFR TKIs in Non-Small Cell Lung Cancer by Activating Src-mediated Bypass Signaling. Clinical cancer research : an official journal of the American Association for Cancer Research 24, 1227-1239 (2018).

40. Ordovas-Montanes J, Rakoff-Nahoum S, Huang S, Riol-Blanco L, Barreiro O, von Andrian UH. The Regulation of Immunological Processes by Peripheral Neurons in Homeostasis and Disease. Trends in immunology 36, 578-604 (2015).

41. Pinho-Ribeiro FA, Verri WA, Jr., Chiu IM. Nociceptor Sensory Neuron-Immune Interactions in Pain and Inflammation. Trends in immunology 38, 5-19 (2017).

42. Gao X, Zhang D, Xu C, Li H, Caron KM, Frenette PS. Nociceptive nerves regulate haematopoietic stem cell mobilization. Nature 589, 591-596 (2021).

43. Zhu S, et al. Subchondral bone osteoclasts induce sensory innervation and osteoarthritis pain. The Journal of clinical investigation 129, 1076-1093 (2019).

44. Fukuda T, et al. Sema3A regulates bone-mass accrual through sensory innervations. Nature 497, 490-493 (2013).

45. Pearson J, Dancis J, Axelrod F, Grover N. The sural nerve in familial dysautonomia. Journal of neuropathology and experimental neurology 34, 413-424 (1975). 
46. Maayan C, Bar-On E, Foldes AJ, Gesundheit B, Pollak RD. Bone mineral density and metabolism in familial dysautonomia. Osteoporosis international : a journal established as result of cooperation between the European Foundation for Osteoporosis and the National Osteoporosis Foundation of the USA 13, 429-433 (2002).

47. Prima V, Depoix C, Masselot B, Formstecher P, Lefebvre P. Alteration of the glucocorticoid receptor subcellular localization by non steroidal compounds. The Journal of steroid biochemistry and molecular biology 72, 1-12 (2000).

48. Ratajczak T, Cluning C, Ward BK. Steroid Receptor-Associated Immunophilins: A Gateway to Steroid Signalling. The Clinical biochemist Reviews 36, 31-52 (2015).

49. Ratajczak T. Steroid Receptor-Associated Immunophilins: Candidates for Diverse DrugTargeting Approaches in Disease. Current molecular pharmacology 9, 66-95 (2015).

50. Periyasamy S, Hinds T, Jr., Shemshedini L, Shou W, Sanchez ER. FKBP51 and Cyp40 are positive regulators of androgen-dependent prostate cancer cell growth and the targets of FK506 and cyclosporin A. Oncogene 29, 1691-1701 (2010).

51. Davies TH, Ning YM, Sanchez ER. Differential control of glucocorticoid receptor hormonebinding function by tetratricopeptide repeat (TPR) proteins and the immunosuppressive ligand FK506. Biochemistry 44, 2030-2038 (2005).

52. Kimmins S, MacRae TH. Maturation of steroid receptors: an example of functional cooperation among molecular chaperones and their associated proteins. Cell stress \& chaperones 5, 76-86 (2000).

53. Banerjee A, et al. Control of glucocorticoid and progesterone receptor subcellular localization by the ligand-binding domain is mediated by distinct interactions with tetratricopeptide repeat proteins. Biochemistry 47, 10471-10480 (2008).

54. Ward BK, Mark PJ, Ingram DM, Minchin RF, Ratajczak T. Expression of the estrogen receptor-associated immunophilins, cyclophilin 40 and FKBP52, in breast cancer. Breast cancer research and treatment 58, 267-280 (1999).

55. Gougelet A, et al. Estrogen receptor alpha and beta subtype expression and transactivation capacity are differentially affected by receptor-, hsp90- and immunophilin-ligands in human breast cancer cells. The Journal of steroid biochemistry and molecular biology 94, 71-81 (2005).

56. Nair SC, Toran EJ, Rimerman RA, Hjermstad S, Smithgall TE, Smith DF. A pathway of multi-chaperone interactions common to diverse regulatory proteins: estrogen receptor, Fes tyrosine kinase, heat shock transcription factor Hsf1, and the aryl hydrocarbon receptor. Cell stress \& chaperones 1, 237-250 (1996). 
57. Ernst K, Kling C, Landenberger M, Barth H. Combined Pharmacological Inhibition of Cyclophilins, FK506-Binding Proteins, Hsp90, and Hsp70 Protects Cells From Clostridium botulinum C2 Toxin. Frontiers in pharmacology 9, 1287 (2018).

59. Ernst K, et al. Pharmacological Cyclophilin Inhibitors Prevent Intoxication of Mammalian

60. Lang AE, et al. The chaperone Hsp90 and PPIases of the cyclophilin and FKBP families facilitate membrane translocation of Photorhabdus luminescens ADP-ribosyltransferases. Cellular microbiology 16, 490-503 (2014).

61. Schuster M, et al. The Hsp90 machinery facilitates the transport of diphtheria toxin into human cells. Scientific reports 7, 613 (2017).

62. $\mathrm{Yu} \mathrm{H}$, et al. The role of aryl hydrocarbon receptor in bone remodeling. Progress in biophysics and molecular biology 134, 44-49 (2018).

63. Herlin M, et al. New insights to the role of aryl hydrocarbon receptor in bone phenotype and in dioxin-induced modulation of bone microarchitecture and material properties. Toxicology and applied pharmacology 273, 219-226 (2013).

64. Iqbal J, et al. Smoke carcinogens cause bone loss through the aryl hydrocarbon receptor and induction of Cyp1 enzymes. Proceedings of the National Academy of Sciences of the United States of America 110, 11115-11120 (2013).

65. Jeong YT, et al. Osteoprotective Effects of Polysaccharide-Enriched Hizikia fusiforme Processing Byproduct In Vitro and In Vivo Models. Journal of medicinal food 19, 805-814 (2016).

66. Hotokezaka H, et al. U0126 and PD98059, specific inhibitors of MEK, accelerate differentiation of RAW264.7 cells into osteoclast-like cells. The Journal of biological chemistry 277, 47366-47372 (2002). 


\section{Figure Legends}

\section{Fig1. Sensory hypersensitivity induces osteopenia in mice}

(a) Sensory activation was assessed by hot plate latency.

(b) Quantitative analyses of bone mineral density (BMD), bone volume/tissue volume (BV/TV), trabecular separation (Tb.Sp), and trabecular number ( Tb.N) of tibia by micro-computed tomography $(\mu \mathrm{CT})$.

(c) Von Kossa staining and quantitative analysis of osteoid volume per tissue volume (OV/TV) and mature bone volume per tissue volume (mTV/BV) in femoral bone tissue.

(d) Representative images of calcein double labeling of trabecular bone of femurs with quantification of mineral apposition rate (MAR), and bone formation rate per bone surface (BFR/BS).

(e) Toluidine blue staining and quantitative analysis of the number of osteoblasts per trabecular bone perimeter (N.Ob/B.Pm).

(f) Maximal loading of femur by three-point bending assay.

(g) ELISA analysis of serum CTX and P1NP levels.

(h) Serum inorganic phosphorus concentration by phosphomolybdate method.

(i-l) Isolated co-culture of SN and osteoclasts. (i) Graphic illustration of isolated co-culture system. (j) Representative Trap staining pictures. Trap+ osteoclasts with more than three nuclei were quantified using Image $\mathrm{J}$ software. (k) Resorption activity was measured by plating cells on fluoresceinated calcium phosphate-coated plates. (l) Expression of the resorption-related genes Mmp9 and Ctsk in osteoclasts.

$* \mathrm{P}<0.05,{ }^{* *} \mathrm{P}<0.01,{ }^{*} * * \mathrm{P}<0.001$, versus controls, Student's $\mathrm{t}$ test. The results are expressed as the mean \pm s.d.

\section{Fig 2. Signals from sensory nerve into bone}

$(a, b)$ Retrograde identification of signals from sensory nerve to bone. Schematic of AAV2-EGFP injection into the tibia (a). After 21 days, DRG was harvested for immunofluorescence staining(b). (c-f) Anterograde identification of signals from sensory nerve to bone. (c) Schematic of VSVEGFP injection into the DRG (L3 and L4). After 5 days, femur was harvested for immunofluorescence staining (d). Confocal images (30- $\mu \mathrm{m}$ z-series) were projected at $1 \mu \mathrm{m}$ intervals to obtain the spatial-localization of EGFP and nucleus. Scatter gram (e) and Pearson's colocalization (f) coefficients were acquired by Imaris X64 software. $(n=5)$

(g-i) Tail vein injection of PHP.S-EGFP (g). After 21 days, tibia was harvested for immunofluorescence staining (h). Image J was used to analyze the confocal images. Graphpad Prism5 was used to analyze the portions. $(\mathrm{n}=5)$ $* \mathrm{P}<0.05,{ }^{* *} \mathrm{P}<0.01,{ }^{* * *} \mathrm{P}<0.001$, and N.S. means not significant, versus controls, Student's $\mathrm{t}$ test. The results are expressed as the mean \pm s.d. 
Fig 3. Cyp40 is crucial in sensory nerve elevating osteoclastogenesis

(a-d) Screening secreted proteins in sensory nerves. (e) Graphic illustration of grouping for iTRAQ. (b) Differential proteins between Y1 and Y2. Red dots indicate proteins that were more abundant in the Y2 group (141 proteins) while green dots represent proteins that are more abundant in the Y1 group (237 proteins). (c) COG function classification of proteins more abundant in Y2, which were considered to be axoplasmic proteins. (d) Saphenous nerve axoplasm proteins related to proliferation and differentiation were identified. These include COF2, TPPP3, AACS, PEDF, GLRX3, PRDX2, UCHL1, GPX1, ADT2, MTPN, MIF, CRYAB, CYP40, MK03, PGK1, and DDB1.

(e) Immunofluorescent staining of identified saphenous nerve axoplasm proteins (Cyp40, Mif, Cf12, Tppp3).

(f, h) Trap staining of osteoclasts, and Trap-positive multiple nucleated cells with $\geq 3$ nuclei per well were scored $(n=3)$.

(g, i) Resorption activity was measured by plating BMMCs on fluoresceinated calcium phosphatecoated plates.

These experiments were repeated in 3 independent biological replicates, each with 3 technical replicates. ${ }^{*} \mathrm{P}<0.05, * * \mathrm{P}<0.01, * * * \mathrm{P}<0.001$, and N.S. means not significant, versus controls, Student's $\mathrm{t}$ test. The results are expressed as the mean \pm s.d.

\section{Fig 4. Cyp40 is a secreted factor, involved in the regulation of sensory nerves on osteoclasts}

(a) Representative confocal images and quantitative analysis of the DRG from the mice injected with RTX or vehicle solution (Ctrl).

(b) ELISA analysis of serum Cyp40 levels.

(c) Confocal images of osteoclasts isolated co-cultured with SN transfected with plasmids containing EGFP-tagged Cyp40.

(d) ELISA analysis of the secreted Cyp40 in the cell culture medium from different numbers of $\mathrm{SN}(\mathrm{n}=3)$.

(e-g) Cyp40 secreted from sensory nerves enters osteoclasts via a non-exosomal mechanism. The co-culture medium was separated into exosome (Exo) and non-exosomal supernatants (MS). Western blot target Cyp40 were performed in Exo and MS (e). (f) Representative IEM images of distribution of EGFP-targeted Cyp40(black granules) in osteoclasts. Osteoclasts on the bone surface were co-cultured with SN transfected with plasmids containing EGFP-tagged Cyp40. (g) Representative IEM images of EGFP-targeted Cyp40 (black granules, red arrow) in brain; membrane vehicles (green arrow).

(h) Western blotting of Ras, c-Raf, Erk, and p-Erk in osteoclasts, which were isolated co-cultured without (Ctrl), or with recombinant Cyp40 (rCyp40), SN (Co-SN), or Cyp40 knock out SN (CoSN (shCyp40)). 
(i) Western blotting of Ras, c-Raf, Erk, and p-Erk in the tibia from the mice injected with RTX or vehicle solution $(\mathrm{Ctrl})$.

(j) NFATc1 mRNA was elevated during sensory nerve-promoted Cyp40-dependent osteoclastogenesis $(n=3)$.

$* \mathrm{P}<0.05, * * \mathrm{P}<0.01, * * * \mathrm{P}<0.001$, and N.S. means not significant, versus controls, Student's $\mathrm{t}$ test. The results are expressed as the mean \pm s.d.

\section{Fig 5. Sensory nerve and its Cyp40 promote osteoclastogenesis by AhR}

(a-d) Osteoclasts were co-cultured with SN transfected with plasmids containing EGFP-tagged Cyp40. (a) Co-immunoprecipitation of EGFP tagged Cyp40 from SN interacts with AhR in osteoclasts.

(b) Western blotting of AhR expression in osteoclasts co-cultured with or without SN.

(c,d) Representative confocal images and analysis of Cyp40-GFP and AhR location in osteoclasts.

(e) Western blotting of AhR, Ras, c-Raf, Erk, and p-Erk in osteoclasts, or AhR knock down osteoclasts (shAhR), or in the modified osteoclasts (shAhR) which has been co-cultured with SN. (f) Trap staining of osteoclasts, and Trap-positive multiple nucleated cells with $\geq 3$ nuclei per well were scored $(n=3)$.

(g) Western blotting of Cyp1a1 in osteoclasts and osteoclasts cultured with SN or recombinant Cyp40.

$* \mathrm{P}<0.05, * * \mathrm{P}<0.01, * * * \mathrm{P}<0.001$, and N.S. means not significant, versus controls, Student's $\mathrm{t}$ test. The results are expressed as the mean \pm s.d.

.


a

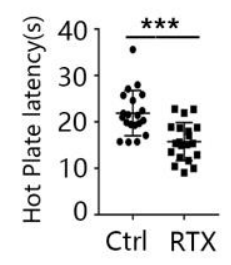

C

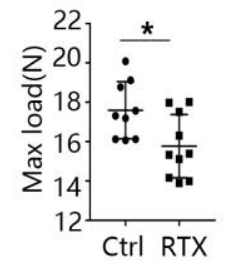

b
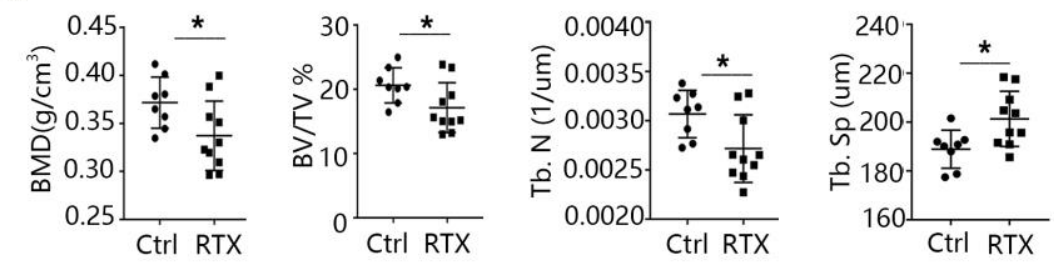

d

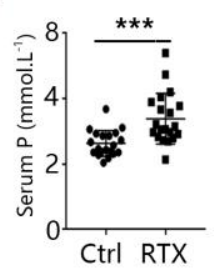

e
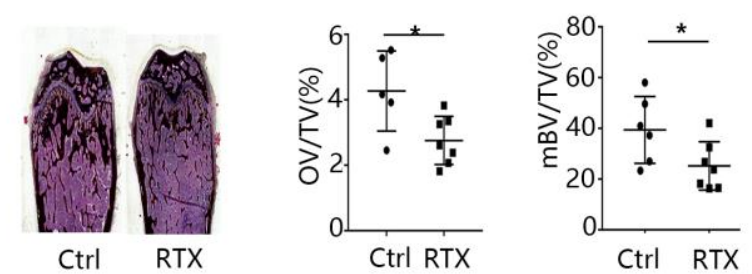

f

Ctrl

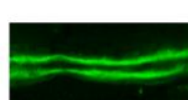

RTX
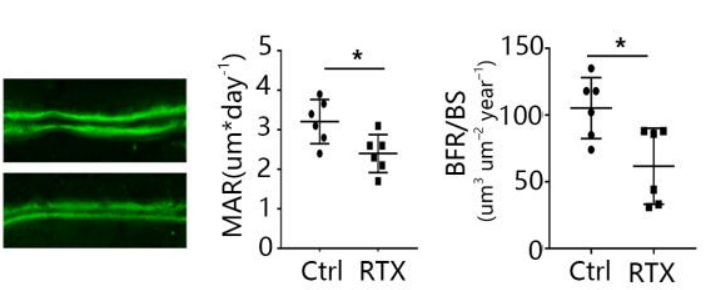

g
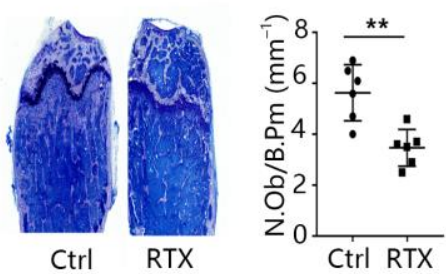

h

i
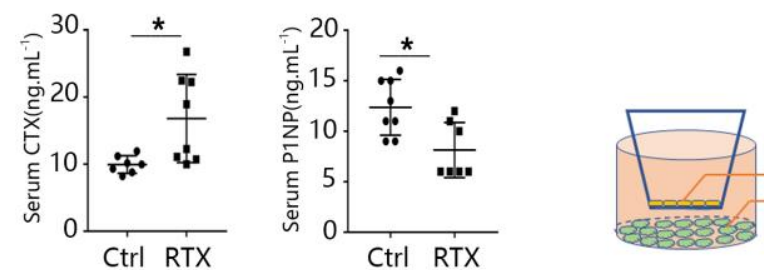

Sensory neurons Osteoclasts
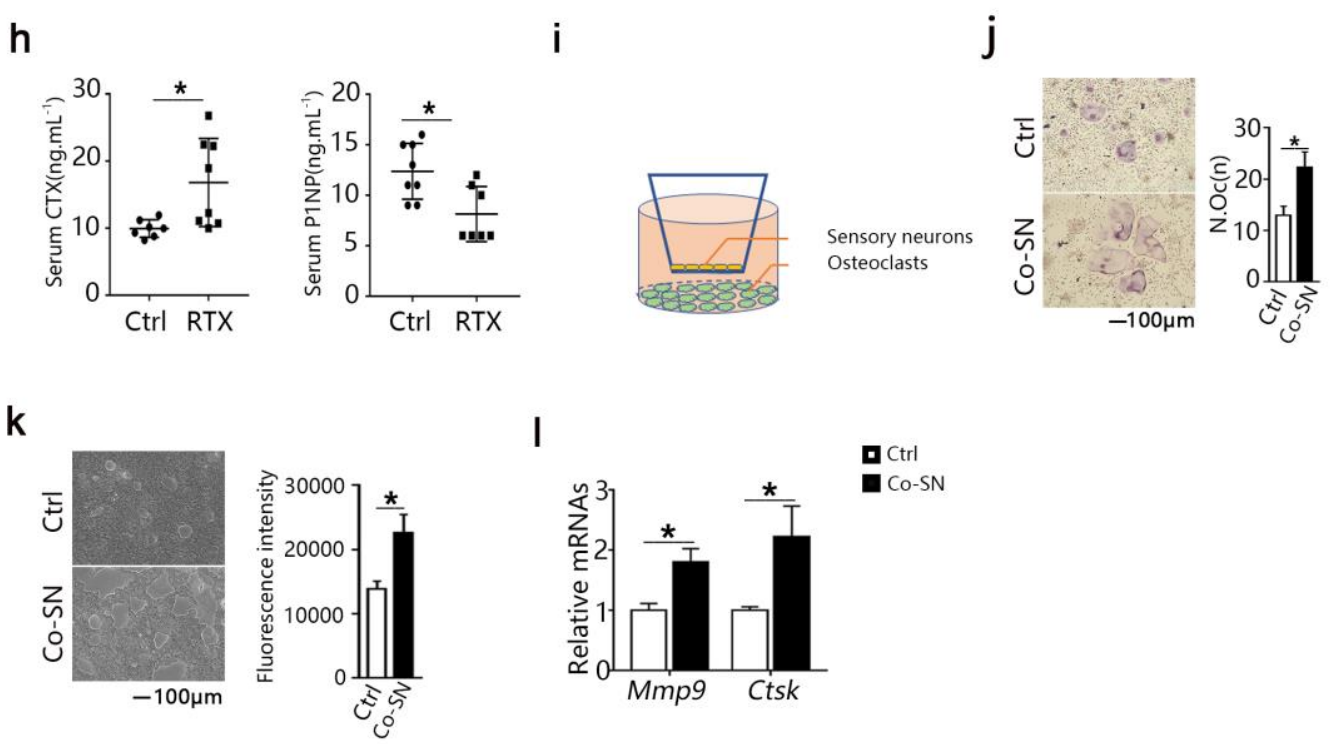
a

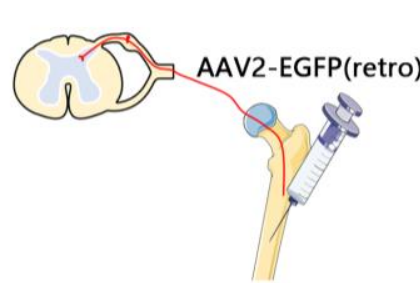

c

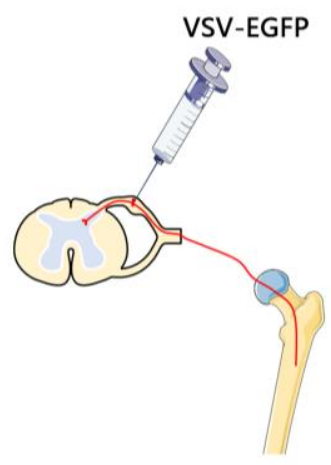

g

PHP.S-EGFP

EQ b

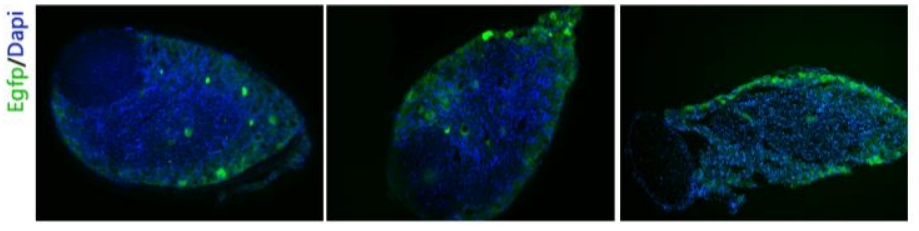

d
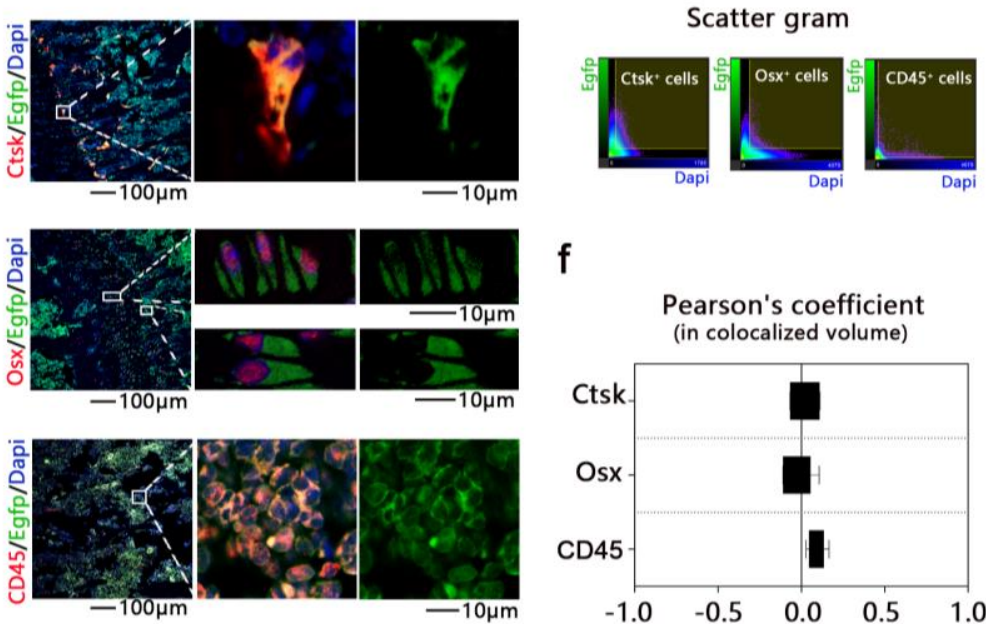

i

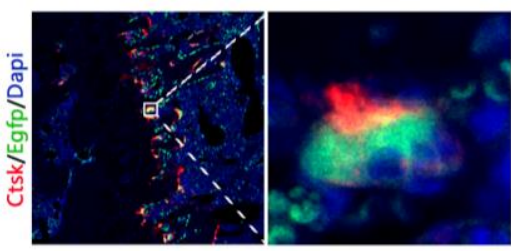

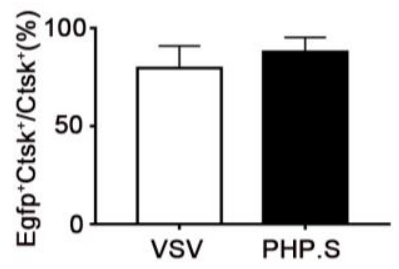


a

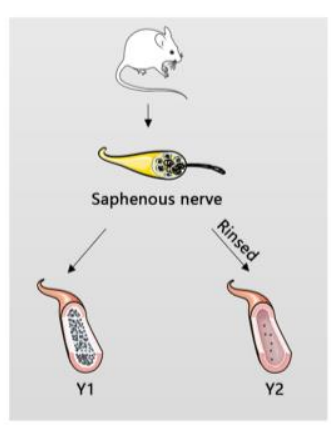

d

Y1_119/Y2_121

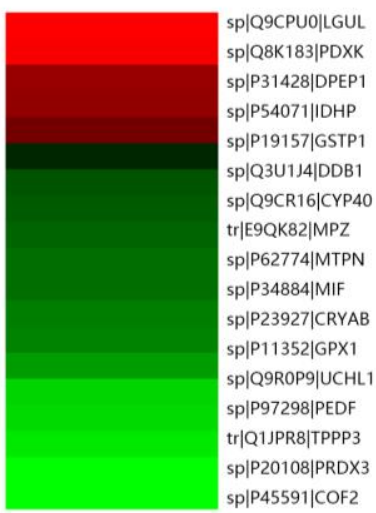

f

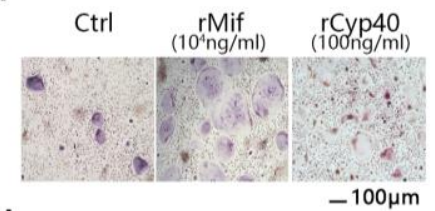

h

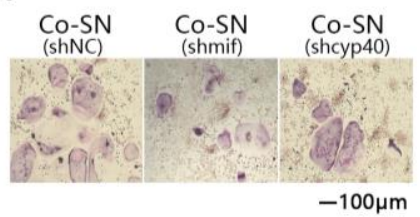

b

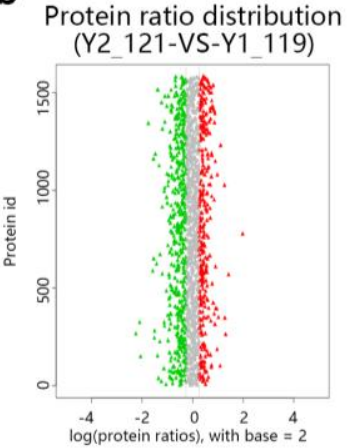

C

COG Function of Mus-Musculus Sequence

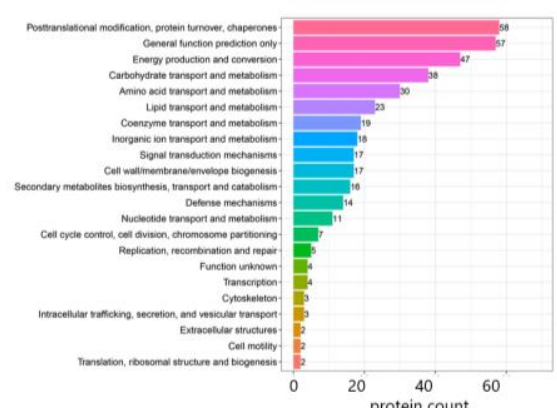

\section{e}
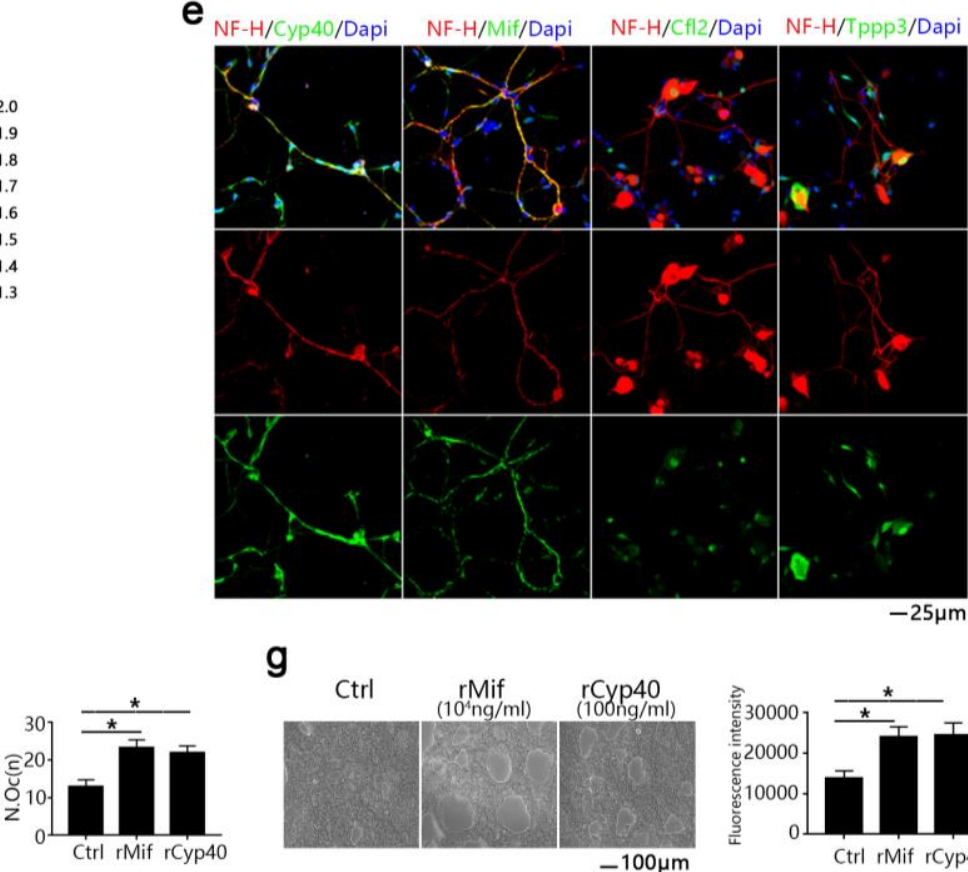

g
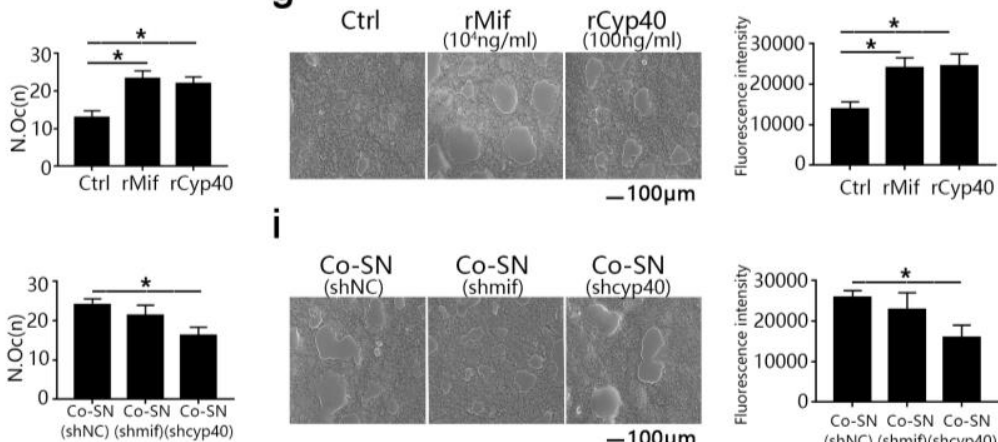

i
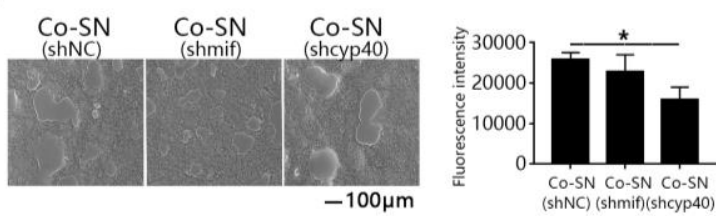
a

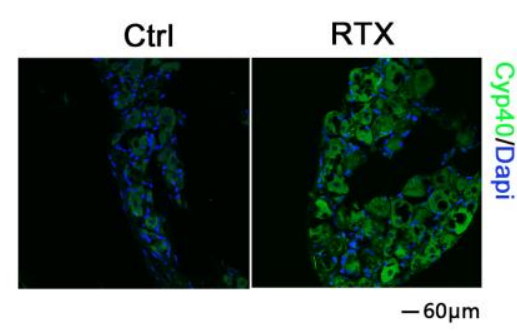

C

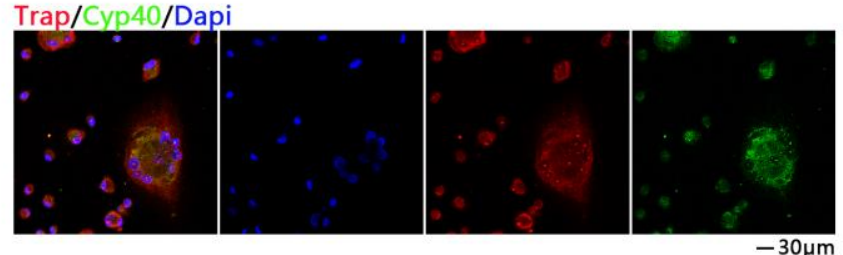

e

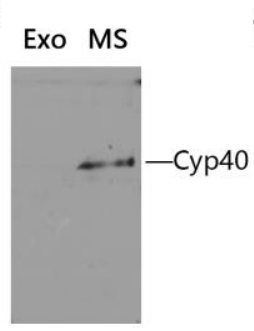

g
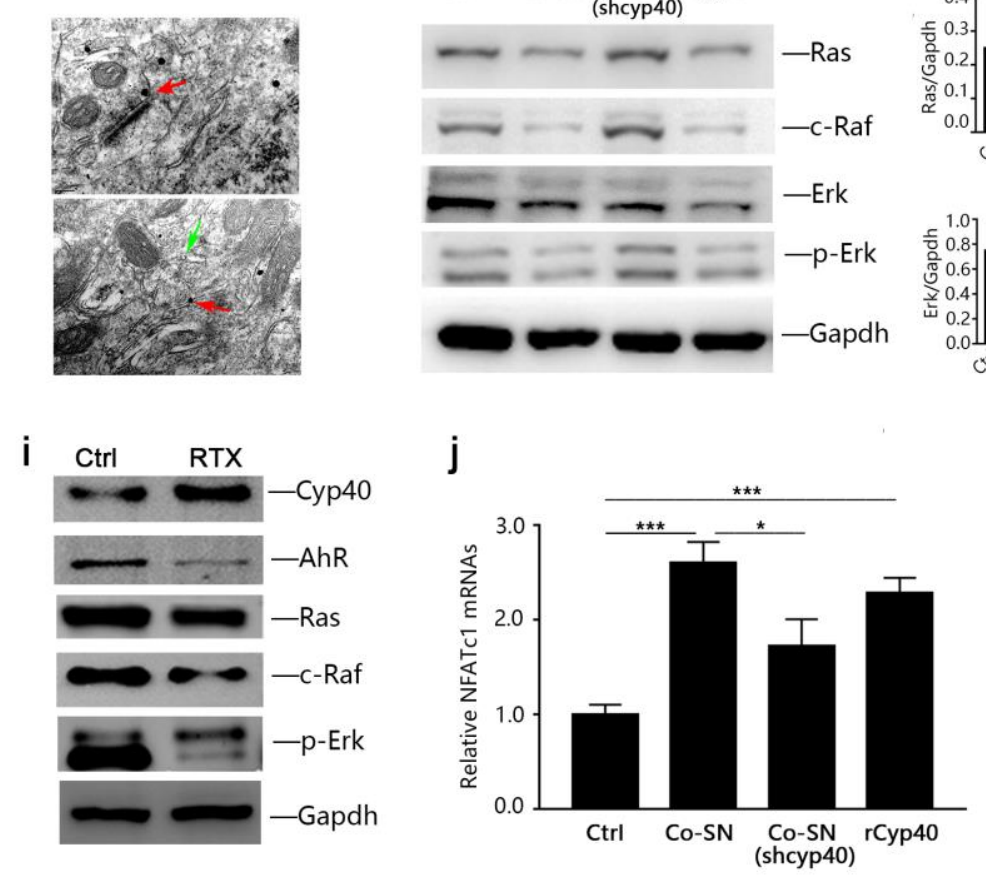

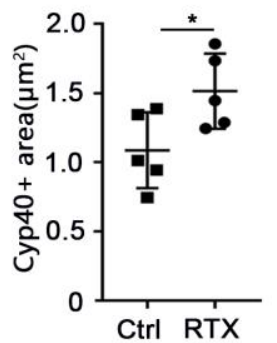

b

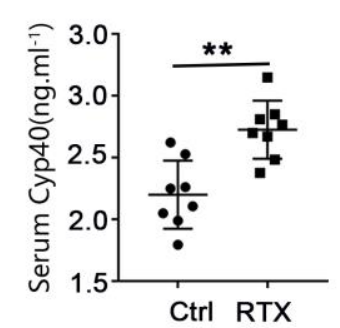

d

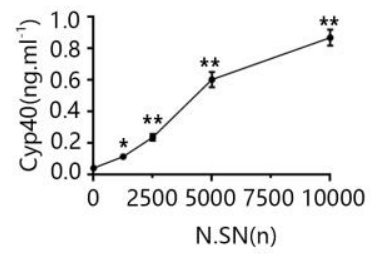

h
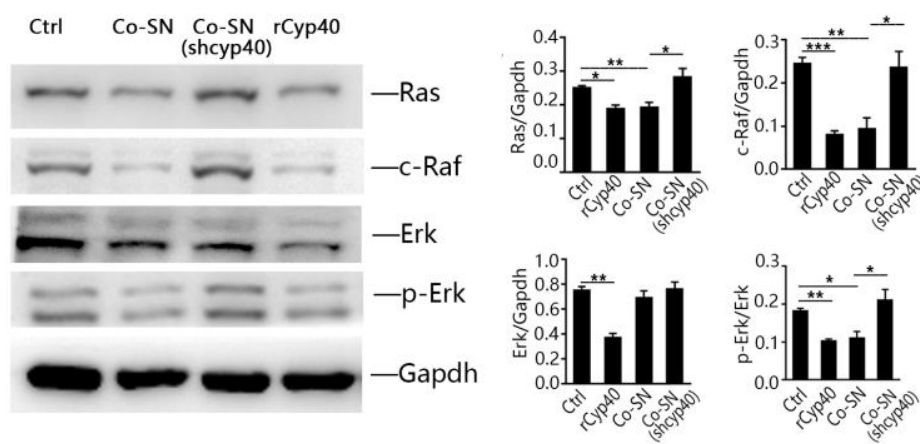
a

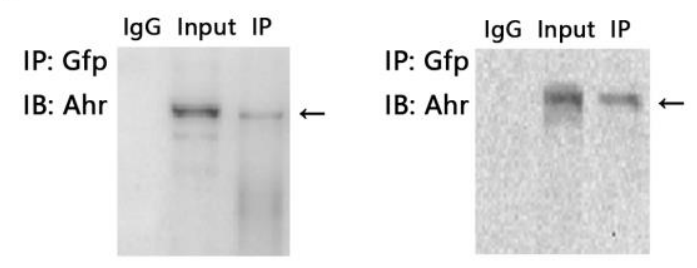

C

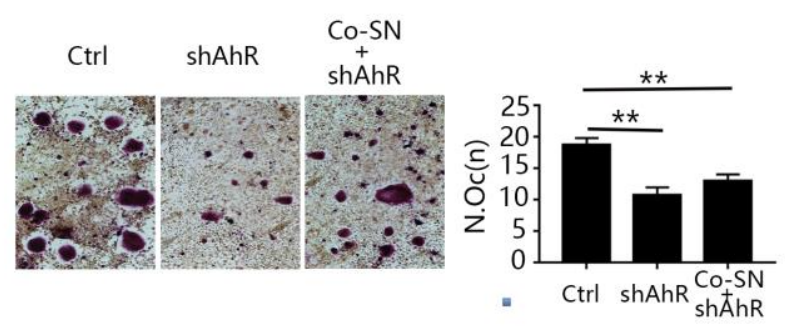

b

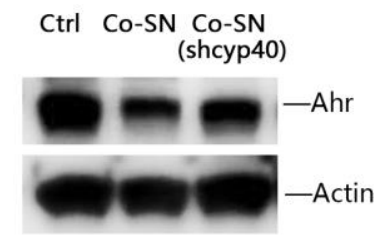

d

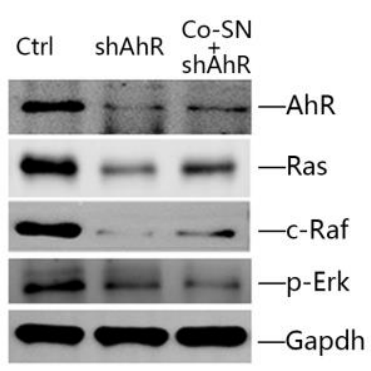

e

AhR/Cyp40/Dapi
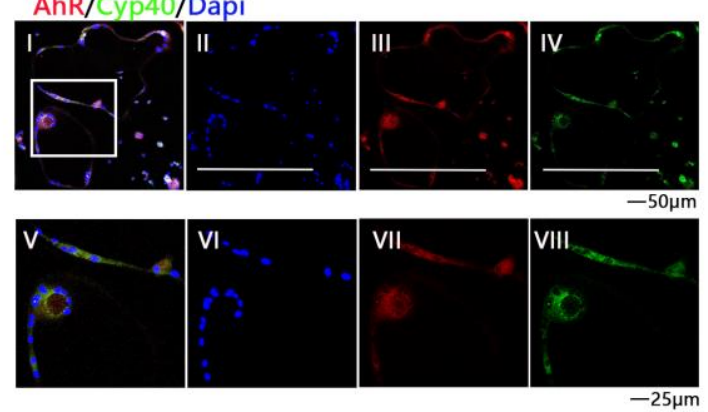

f
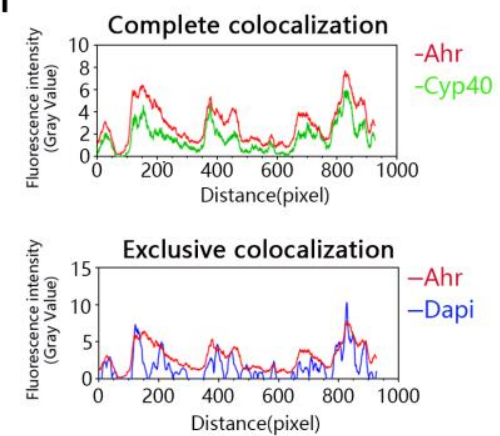

g

Ctrl Co-SN rCyp40

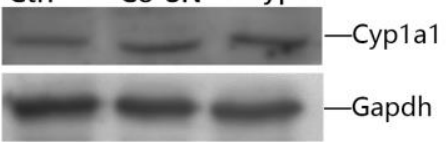




\section{Supplementary Files}

This is a list of supplementary files associated with this preprint. Click to download.

- SupplementMaterials.pdf 